\title{
Relationship Between Palpitation and Mental Health
}

\author{
Fatemeh Alijaniha, ${ }^{1}$ Ahmadali Noorbala,, ${ }^{2 *}$ Suleiman Afsharypuor, ${ }^{3}$ Mohsen Naseri, \\ Faramarz Fallahi, ${ }^{5}$ Mahmood Mosaddegh, ${ }^{6}$ Soghrat Faghih Zadeh, ${ }^{7}$ and Sima Sadrai ${ }^{8}$ \\ ${ }^{1}$ Department of Traditional Pharmacy, School of Traditional Medicine, Shahid Beheshti University of Medical Sciences, Tehran, IR Iran \\ 2 Psychosomatic Ward, Imam Khomeini Hospital, Tehran University of Medical Sciences, Tehran, IR Iran \\ ${ }^{3}$ Faculty of Pharmacy and Pharmaceutical Sciences, Isfahan University of Medical Sciences, Isfahan, IR Iran \\ Faculty of Pharmacy and Pharmaceutical Sciences, Isfahan University of Medical Scien
4 Traditional Medicine Clinical Trial Research Center, Shahed University, Tehran, IR Iran \\ ${ }^{5}$ Traditional Medicine Clinical Trial Research Center, Shahed
Cardiology Department, Shahed University, Tehran, IR Iran \\ 6 Dean Traditional Medicine and Materia Medica Research Center (TMRC), Shahid Beheshti University of Medical Sciences, Tehran, IR Iran \\ ${ }_{8}^{7}$ Department of Biostatistics and Epidemiology, School of Medicine, Zanjan University of Medical Sciences, Zanjan, IR Iran \\ 8 Division of Biopharmaceutics and Pharmacokinetics, Department of Pharmaceutics, Faculty of Pharmacy, Tehran University of Medical Sciences, Tehran, IR Iran \\ ${ }^{*}$ Corresponding Author: Ahmadali Noorbala, Psychosomatic Ward, Imam Khomeini Hospital, Tehran University of Medical Sciences, Tehran, IR Iran. Tel: +98-261192421, Fax: \\ +98-2166930330, E-mail: Noorbala1@gmail.com
}

Received 2014 August 9; Revised 2014 September 10; Accepted 2014 September 29.

\begin{abstract}
Background: 'Palpitation' is one of the most common complaints in patients referring to cardiologists. In modern medicine era, these patients suffer from much distress and some cases are known to be difficult to treat. Although the clinician's first duty is obviously to search for an organic basis for this symptom, the diagnostic evaluation is frequently unrevealing. However, clinical experience suggests that psychiatric causes are relatively common.

Objectives: This research aimed to screen for mental disorders in patients complaining of palpitation and healthy persons in order to perform a preliminary comparison between them.

Patients and Methods: This is a case-control study to screen mental disorders. The target population consisted of adult volunteers with benign palpitation and their matched healthy persons. They were referred during a 10-month-period to the cardiology outpatient's clinic of Mostafa Khomeini hospital in Tehran, Iran. Sampling was accidental and eventually 110 participants comprised the sample size. The measuring tool was GHQ-28 (28-item general health questionnaire) and the main variable was the questionnaire score obtained from the Likert scoring method.

Results: Comparing two groups showed that the number of participants with the scores more than cut-off point in palpitation group was significantly more than healthy person group ( $85.4 \%$ vs. $43.6 \%$ with $\mathrm{P}<0.001)$. Also the total score of GHQ-28 and scores of its subscale (somatization, anxiety, and social dysfunction) in patients complaining of palpitation were significantly more than those of the healthy participants (34.2 vs. $25.7,8.9$ vs. 6.4, 9.4 vs. 6.4, and 12.3 vs.10.8, respectively with $\mathrm{P}<0.001, \mathrm{P}=0.001, \mathrm{P}<0.001$, and $\mathrm{P}<0.007$, respectively). Conclusions: Palpitation is the most common symptom in psychiatric disorders such as anxiety and somatization disorders. According to the results of this study, psychiatric causes have an important role in Iranian patients complaining of palpitations (benign form). Considering this fact may lead to a more effective treatment of benign palpitations.
\end{abstract}

Keywords: Anxiety, Mental Health, Palpitation, Somatization

\section{Background}

Palpitation, defined as an unpleasant awareness of the beating of the heart, is one of the most common complaints in patients referring to cardiac clinics (11\% - 40\%) (1-4). The etiology of palpitation determined by a study was cardiac in $43 \%$, psychiatric in $31 \%$, miscellaneous in $10 \%$, and unknown in $16 \%$ of cases (4). Although some palpitations may be an indication of life-threatening cardiac arrhythmias, most of them are benign without any serious underlying heart disease (5). Benign palpitations, which are generally considered as palpitations without any more serious underlying heart disease, despite its good prognosis, causes considerable distress and disability among affected patients (6).
Despite its high prevalence, in many cases, the diagnostic and therapeutic management of this symptom proved to be poorly efficacious and lead to permanent distress and disability in patients and continual recourse to healthcare facilities (7). One study has shown that $84 \%$ of patients still reported palpitations after 6-month follow-up consultation (8). Today, most research has focused on other cardiovascular patients, whereas less attention has been given to those with palpitation (1). There is some evidence of an association between psychiatric disorders and a subsequent poor outcome in patients suffering from palpitation (1). Palpitations are one of the DSM-III-R diagnostic criteria for

Copyright (C) 2016, Iranian Red Crescent Medical Journal. This is an open-access article distributed under the terms of the Creative Commons Attribution-NonCommercial 4.0 International License (http://creativecommons.org/licenses/by-nc/4.0/) which permits copy and redistribute the material just in noncommercial usages, provided the original work is properly cited. 
panic attack, somatization disorder, and generalized anxiety disorder (4). Although some studies indicated the strong correlation of cardiovascular symptoms to emotional distress (9), to the best of our knowledge, no screening of Iranian patients with benign palpitations for psychiatric disorders has been performed so far. Thus, there is a need for an evaluation of the mental health of these patients, and this study aimed to evaluate the mental health condition in these patients in order to compare it with their matched healthy persons.

\section{Objectives}

This study was done to evaluate the relationship between palpitation symptom and mental health by comparing the mental health status of patients suffering from benign palpitations to their matched healthy persons. Thus, after separating patients suffering from benign palpitations from those with dangerous ones, using current diagnostic strategies $(2,10,11)$, the comparison of the mental health condition between groups was done.

\section{Patients and Methods}

This study used accidental sampling method. Eligible participants were volunteers who were adult outpatients with palpitation (an unpleasant sensation in the heart or awareness of heartbeat) as their main complaint, as well as the matched group of healthy volunteers. Volunteers were called through advertisement. Patients were initially screened by a short interview on the phone to determine palpitation characteristics in order to take a brief record of each patient's medical history, including their health problems and medications to check the inclusion criteria.

Inclusion criteria were as follows: having palpitations (abnormal awareness of heartbeat) as the main complaint for at least three months; aged between 18 and 60 years; and giving their informed consent. Volunteers who met at least one exclusion criterion were not included in the trial. Exclusion criteria were as follows: having mental retardation, psychosis, or other serious psychiatric disorders confirmed by a psychiatrist; having organic heart diseases confirmed by a cardiologist; and suffering from serious chronic diseases. After primary selection of the eligible patients, they were invited to the outpatient cardiac clinic of Mostafa Khomeini hospital. It is a general hospital with 280 beds and 9 hospital sections, which is a semi-governmental hospital affiliated to Shahed university. Each patient's medical history was checked by a cardiologist, and each had a physical examination, an electrocardiography test, and biochemical laboratory tests to identify those patients suffering from serious problems considered under the exclusion criteria.

Patients' mental health was evaluated and they were screened for probable disorders by means of GHQ-28. Furthermore, healthy participants selected from accessible volunteers working in telecommunication office, non- governmental companies or were housekeepers, of similar age, gender, and educational degree, were included in the study. They were also evaluated for mental health and screened for probable disorders by means of GHQ-28.

An Iranian version of the 28-item general health questionnaire (GHQ-28) was used as the measuring tool. It is a brief, simple, consistent and reliable instrument, and one of the most well-known questionnaires for the detection of mental disorders. This questionnaire was developed by Goldberg and Hillier (1979) for screening somatic symptoms, anxiety, insomnia, social dysfunction, and severe depression (12). It has been translated into 38 different languages (13) as well as the official language of Iran (Persian) and its validity and reliability were approved in an independent study (14). Also, other studies on the validation of the GHQ-28 in different countries demonstrate its high validity and reliability as a screening tool of mental disorders in the community $(15,16)$.

The study used the Likert scoring method, in which the maximum score was 84 . The cut-off point was 23 out of the total score and 6 in every subscale; thus, patients with the equal or higher than cut-off point scores were deemed as having a psychological disorder. Sensitivity, specificity, and overall misclassification rate for a GHQ28 cut-off score of 6 were 84.7, 93.8, and 8.2\%, respectively. The reliability of the GHQ-28 was assessed in a sample of 90 participants retested 1 week after the initial referral. The estimated intraclass correlation between the testretest scores was 0.85 (17).

Each item is accompanied by four possible responses of typically 'not at all,' 'no more than usual,' 'rather more than usual,' and 'much more than usual,' scoring from 0 to 3 , respectively.

The total possible score on the GHQ-28 ranges from 0 to 84 and allows for means and distributions to be calculated, both for the global total, as well as for the 4 sub-scales (18).

This study was performed alongside with a clinical trial, which was approved by Medical Ethics Committee of Shahid Beheshti University of Medical Sciences (accession No. 111, 2012 - 1 - 7). Regarding the ethical consideration, all participants provided written informed consent; they could ask any question and leave the study whenever they wish, also data privacy was considered. According to previous data from pilot and other studies and considering: $\alpha=0.05, \beta=0.20, \sigma=12$, and $\Delta=7$, the sample size was calculated by the following formula as at least 46 in every group (Equation 1). So total sample size of 110, was considered suitable for compensating the probable exclusion of some participants.

$$
\mathrm{n}=\left(\mathrm{r}+\frac{1}{\mathrm{r}}\right) \sigma^{2} \frac{\left(\mathrm{Z}_{\beta}+\mathrm{Z}_{\frac{\alpha}{2}}\right)^{2}}{\Delta^{2}}
$$

Using SPSS software (version 20), normal distribution was checked by Kolmogorov-Smirnov test. Independent 
samples t test was performed for scores of questionnaire due to normal distribution. The Chi-square test was applied to compare demographic data. It also used for comparing the number of patients with scores more than the cut-off point between the groups due to lack of normal distribution. Data are presented as means \pm standard deviations (SD) and proportions (\%). Differences were considered significant with $\mathrm{P}<0.05$.

\section{Results}

Out of 109 primary volunteer patients, 55 were included in this study. Thirty-eight patients excluded because of refusing to participate or not meeting inclusion criteria and 16 patients excluded because of their incomplete questionnaires. In addition, 55 healthy volunteers who were matched to patients group considering their age, sex, and degree of education, were included. The mean age of participants was $40.1 \pm 11.3 \mathrm{y}$; and $63.6 \%$ of them were female (Table 1 ). Their age ranged from 18 to $60 \mathrm{y}$. The most common educational degree was diploma (39.1\%).

There was no significant difference with respect to age, gender, and degree of education between the two groups (patients and healthy volunteers) (Table 1).

Results showed that, 24 out of 55 participants (43.6\%) in the healthy persons group and 47 out of 55 participants (85.4\%) in the patients group did not have a proper state of mental health (according to the scores that were above the cut-off point). There is a significant difference between the groups $(\mathrm{P}<0.001)$.

According to the four subscales of mental health, as shown in Table 2, the most prevalent disorder was social dysfunction and the least prevalent one was depression. The rate of disorder with respect to somatization and anxiety in patients with palpitation was significantly more than healthy participants.

As shown in Table 3, the total scores of GHQ-28 and 3 out of 4 of its subscales, including somatization, anxiety, and social dysfunction, in patients complaining of palpitation were significantly more than healthy participants (34.2 vs. 25.7, $\mathrm{P}<0.001 ; 8.9$ vs. $6.4, \mathrm{P}=0.001 ; 9.4$ vs. $6.4, \mathrm{P}$ $<0.001$; and 12.3 vs. 10.8, $\mathrm{P}<0.007$; respectively). Regarding depression subscale, the difference of two groups was not significant $(\mathrm{P}=0.089)$.

Table 1. Comparison Between Two Groups; Patients With Heart Palpitation and Healthy Persons ${ }^{\mathrm{a}, \mathrm{b}}$

\begin{tabular}{lccc}
\hline Demographic Data & Healthy Persons & Patients With Palpitation & P Value \\
\hline Age, $\mathbf{y}$ & $38.4 \pm 11.3$ & $41.8 \pm 11.4$ & .116 \\
Gender & $35(63.6)$ & $35(63.6)$ & $>.999$ \\
Educational level, $\mathbf{y}$ & $13.7 \pm 3.6$ & $13.2 \pm 2.8$ & .195 \\
\hline
\end{tabular}

$\mathrm{a}_{\mathrm{N}}=55$.

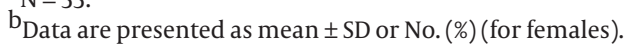

Table 2. Frequency of Participants With Disorder of Mental Health According to Subscales of GHQ-28

\begin{tabular}{|c|c|c|c|}
\hline Aspect of Mental Health & Number of Patients With Disorder & Percentage of Participants With Disorder & P Value \\
\hline Total score & & & $<.001$ \\
\hline Patients with palpitation & 47 & 85.4 & \\
\hline Healthy persons & 24 & 43.6 & \\
\hline Somatization & & & $<.001$ \\
\hline Patients with palpitation & 45 & 81.8 & \\
\hline Healthy persons & 23 & 41.8 & \\
\hline Anxiety & & & $<.004$ \\
\hline Patients with palpitation & 44 & 80 & \\
\hline Healthy persons & 30 & 54.6 & \\
\hline Social dysfunction & & & $<.081$ \\
\hline Patients with palpitation & 53 & 96.4 & \\
\hline Healthy persons & 48 & 87.2 & \\
\hline Depression & & & $<.238$ \\
\hline Patients with palpitation & 13 & 23.6 & \\
\hline Healthy persons & 9 & 16.4 & \\
\hline
\end{tabular}


Alijaniha F et al.

\begin{tabular}{lccc}
\hline Table 3. GHQ-28 Scores of Two Groups & \\
\hline Variable & & \\
\hline b & Patients With Palpitation & Healthy Persons & P Value \\
\hline Total score & $34.29(11.6)$ & $25.7(11.6)$ & $<.001$ \\
Somatization subscale & $8.96(3.5)$ & $6.42(4.4)$ & .001 \\
Anxiety subscale & $9.45(4.3)$ & $6.43(3.7)$ & $<.001$ \\
Social dysfunction subscale & $12.38(2.5)$ & $10.80(3.4)$ & .007 \\
Depression subscale & $3.49(11.6)$ & $2.10(3.3)$ & .089 \\
\hline
\end{tabular}

${ }^{\mathrm{a}}$ Data are presented as mean (SD).

${ }^{\mathrm{b}}$ Data are presented for GHQ score.

\section{Discussion}

Although palpitation is generally considered as a cardiovascular disease, some studies showed that more than $50 \%$ of the patients with a complaint of palpitation had no heart disease (19) instead usually suffer from psychiatric disorders (1, 20-23). Clinical experiments have shown that psychiatric causes are prevalent in patients with palpitation and the most common form of these problems are anxiety, depression, and somatoform disorders. These patients experience frequent referrals to cardiology clinics and $10 \%-14 \%$ of them have panic disorders (4). As indicated in a study by Barsky et al. about half of the patients with palpitation evaluated by a 24-hour ECG or event recorder, had suffered from psychiatric disorders (24).

However, our findings indicated that $85.4 \%$ of the patients with palpitation had psychiatric disorders and the frequency of mental disorder in them was more than similar group of healthy persons (about twice). Moreover, according to this result, the prevalence of mental disorder (43.6\%) in healthy group was more than the results of an extensive study implemented in Iran. That survey, which had a large sample size, had reported that $34.2 \%$ of the population in the age group of 15 years and above suffer from mental disorders (25).

Our findings showed that among the four aspects of mental health evaluated by the GHQ-28, social dysfunction was the most prevalent disorder; however, there is no significant difference between patients with palpitation and healthy participants in this regard. On the other hand, somatization and anxiety had significantly more prevalence in patients with palpitation compared to healthy participants and depression had the least prevalence and did not show any significant difference between the groups.

Jonsbu et al. reported the prevalence of psychiatric disorders in patients with benign palpitation as follows: anxiety disorder 30\%, major depression $4 \%$, and any somatoform disorder 15\% (1). Likewise, our findings showed that depression had the least prevalence, however, the prevalences of anxiety, major depression, and somatization were $81.8,23.6$, and $81.8 \%$, respectively.

In some studies, anxiety is considered as the most common non-cardiac cause of palpitation (26). Some others suggested that panic attacks and somatization are closely related to it $(4,5,27-30)$. Furthermore, depression is known as a common cause of palpitation (27, 31-35). Our findings also indicated that anxiety was the most common disorder in patients, considering it as one of two disorders that were significantly more in patients than healthy persons.

To the best of our knowledge, this is the first study examining Iranian patients with palpitation compared to similar healthy persons; however, as a preliminary evaluation, the use of a screening tool can be considered as a limitation and a clinical psychiatric evaluation is proposed for the next study.

As mentioned before, palpitation is one of the most common symptoms that prompt patients to consult a cardiologist. However, most of the times, it is considered benign and without any cardiac origin. It seems that palpitation is associated with psychiatric symptoms such as anxiety and somatization. Therefore, in psychiatric disorders with symptoms of anxiety and somatization, palpitation is very common. Since the accurate etiology leads to an effective treatment, it is highly recommended that patients with palpitation after a negative evaluation by a cardiologist, be referred for psychiatric assessments.

\subsection{Strong Points of Study}

Given the considerable prevalence of palpitation and lack of effective treatment from cardiac point of view, considering a psychiatric origin after rolling out the cardiac underlying causes, may lead to more suitable treatments. Besides, to the best of our knowledge this study is the first one, which evaluates the relationship between palpitation and mental health.

\subsection{Weak Points of Study}

GHQ-28 is a screening tool and in order to evaluate more accurately psychiatric disorders such as anxiety, depression, and so on, specific questionnaires for each of them may be necessary, as well as a clinical psychiatric evaluation and a bigger sample size that lead to more statistically valid conclusions.

\section{Acknowledgments}

The article was based on a part of PhD thesis of traditional pharmacy (Fatemeh Alijaniha, No. 127), granted by 
the school of traditional medicine, Shahid Beheshti university of medical sciences.

\section{Footnotes}

Authors' Contribution:Study concept and design: Ahmadali Noorbala and Fatemeh Alijaniha; acquisition of data, analysis and interpretation of data, drafting of the manuscript: Fatemeh Alijaniha; critical revision of the manuscript for important intellectual content: Ahmadali Noorbala, Mohsen Naseri, Faramarz Fallahi, Suleiman Afsharypuor, and Sima Sadrai; statistical analysis: Soghrat Faghih Zadeh and Sima Sadrai; administrative, technical, and material support: Ahmadali Noorbala and Mahmood Mosaddegh; and Study supervision: ahmadali Noorbala.

Financial Disclosure:The funding organization had no role in the design and conduct of the study; nor collection, management, and analysis of the data; or preparation, review, and approval of the manuscript.

Funding/Support:This research project was sponsored by the school of traditional medicine, Shahid Beheshti university of medical sciences, Tehran, Iran.

\section{References}

1. Jonsbu E, Dammen T, Morken G, Martinsen EW. Patients with noncardiac chest pain and benign palpitations referred for cardiac outpatient investigation: a 6-month follow-up. Gen Hosp Psychiatry. 2010;32(4):406-12. doi: 10.1016/j.genhosppsych.2010.03.003. [PubMed: 20633745]

2. Khamis RY, Dancy M. Palpitations. Medicine. 2009;37(2):100-5. doi:10.1016/j.mpmed.2008.11.012.

3. Summerton N, Mann S, Rigby A, Petkar S, Dhawan J. New-onset palpitations in general practice: assessing the discriminant value of items within the clinical history. Fam Pract. 2001;18(4):38392. [PubMed: 11477045]

4. Weber BE, Kapoor WN. Evaluation and outcomes of patients with palpitations. Am J Med.1996;100(2):138-48. [PubMed: 8629647]

5. Abbott AV. Diagnostic approach to palpitations. Am Fam Physician. 2005;71(4):743-50. [PubMed: 15742913]

6. Price JR. Medically unexplained physical symptoms. Medicine. 2008;36(9):449-51. doi:10.1016/j.mpmed.2008.06.006.

7. Raviele A, Giada F, Bergfeldt L, Blanc JJ, Blomstrom-Lundqvist C, Mont L, et al. Management of patients with palpitations: a position paper from the European Heart Rhythm Association. Europace. 2011;13(7):920-34. doi: 10.1093/europace/eur130. [PubMed: 21697315]

8. Barsky AJ, Cleary PD, Coeytaux RR, Ruskin JN. The clinical course of palpitations in medical outpatients. Arch Intern Med. 1995;155(16):1782-8. [PubMed: 7654112]

9. Ketterer MW, Knysz W, Keteyian SJ, Schairer J, Jafri S, Alam M, et al. Cardiovascular symptoms in coronary-artery disease patients are strongly correlated with emotional distress. Psychosomatics. 2008;49(3):230-4. doi: 10.1176/appi.psy.49.3.230. [PubMed: 18448778]

10. Kopp DE, Wilber DJ. Palpitations and arrhythmias. Separating the benign from the dangerous. Postgrad Med. 1992;91(1):241-4. [PubMed: 1728776]

11. Taggar JS, Hodson A. The assessment and management of palpitations in primary care. InnovAiT RCGP J Associates Train. 2011;4(7):408-13.

12. Goldberg DP, Hillier VF. A scaled version of the General Health Questionnaire. Psychol Med.1979;9(1):139-45. [PubMed: 424481]

13. Jackson C. The general health questionnaire. Occup Med. 2007;57(1):79.

14. Noorbala AA, Mohammad K. The validation of general health questionnaire-28 as a psychiatric screening tool. Hakim Res J.

\section{9;11(4):47-53.}

15. Ebrahimi AE, Molavi H, Moosavi SG, Bornamanesh AR, Yaghobi M. Psychometric properties and factor structure of general health questionnaire 28 (GHQ-28) in Iranian psychiatric patients. J Res Behav Sci. 2008;5(1):5-12.

16. Noorbala AA, Bagheri Yazdi SA, Yasamy MT, Mohammad K. Mental health survey of the adult population in Iran. Br J Psychiatry. 2004;184:70-3. [PubMed:14702230]

17. Noorbala A, Mohammad K, Bagheri Yazdi S. Validation of GHQ-28 in Iran. Hakim Res J.1999;5(1):101-10.

18. Makowska Z, Merecz D, Moscicka A, Kolasa W. The validity of general health questionnaires, GHQ-12 and GHQ-28, in mental health studies of working people. Int J Occup Med Environ Health. 2002;15(4):353-62. [PubMed:12608623]

19. Jonsbu E, Dammen T, Morken G, Moum T, Martinsen EW. Shortterm cognitive behavioral therapy for non-cardiac chest pain and benign palpitations: a randomized controlled trial. J Psychosom Res. 2011;70(2):117-23. doi: 10.1016/j.jpsychores.2010.09.013. [PubMed: 21262413]

20. Ehlers A, Mayou RA, Sprigings DC, Birkhead J. Psychological and perceptual factors associated with arrhythmias and benign palpitations. Psychosom Med. 2000;62(5):693-702. [PubMed: 11020100]

21. Jonsbu E, Martinsen EW, Morken G, Moum T, Dammen T. Illness perception among patients with chest pain and palpitations before and after negative cardiac evaluation. Biopsychosoc Med. 2012;6(1):19. doi: 10.1186/1751-0759-6-19. [PubMed: 23017128]

22. Mayou R. Chest pain, palpitations and panic. J Psychosom Res. 1998;44(1):53-70. [PubMed: 9483464]

23. Mayou R, Sprigings D, Birkhead J, Price J. Characteristics of patients presenting to a cardiac clinic with palpitation. QJM. 2003;96(2):115-23. [PubMed:12589009]

24. Barsky AJ. Palpitations, cardiac awareness, and panic disorder. Am J Med.1992;92(1A):31S-4S. [PubMed:1734732]

25. Noorbala AA, Bagheri Yazdi SA, Asadi Lari M, Mahdavi V, Reza M Mental health status of individuals fifteen years and older in Tehran-Iran (2009). Iran J Psychiat Clin Psych. 2011;16(4):479-83.

26. Wexler RK, Pleister A, Raman S. Outpatient approach to palpitations. Am Fam Physician. 2011;84(1):63-9. [PubMed: 21766757]

27. Jonsbu E, Dammen T, Morken G, Lied A, Vik-Mo H, Martinsen EW Cardiac and psychiatric diagnoses among patients referred for chest pain and palpitations. Scand Cardiovasc J. 2009;43(4):256-9. doi:10.1080/14017430902946749. [PubMed: 19431049]

28. Schruers KRJ, Van De Mortel H, Overbeek T, Griez E. Symptom profiles of natural and laboratory panic attacks. Acta Neuropsychiat. 2004;16(2):101-6. doi:10.1111/j.0924-2708.2004.0084.x.

29. Thavendiranathan P, Bagai A, Khoo C, Dorian P, Choudhry NK. Does this patient with palpitations have a cardiac arrhythmia? JAMA. 2009;302(19):2135-43. doi: 10.1001/jama.2009.1673. [PubMed:19920238]

30. Thompson J. Psychological and physical etiologies of heart palpitations. Nurse Pract. 2006;31(2):14-7. [PubMed: 16452897]

31. Caballero L, Aragones E, Garcia-Campayo J, Rodriguez-Artalejo F, Ayuso-Mateos JL, Polavieja P, et al. Prevalence, characteristics, and attribution of somatic symptoms in Spanish patients with major depressive disorder seeking primary health care. Psychosomatics. 2008;49(6):520-9. doi: 10.1176/appi.psy.49.6.520. [PubMed: 19122129]

32. Greco T, Eckert G, Kroenke K. The outcome of physical symptoms with treatment of depression. J Gen Intern Med. 2004;19(8):813-8. doi:10.1111/j.1525-1497.2004.30531.x. [PubMed:15242465]

33. Johansson P, Riegel B, Svensson E, Broström A, Alehagen U, Dahlström U, et al. The contribution of heart failure to sleep disturbances and depressive symptoms in older adults. J geriatr Psychiat Neurol. 2012;25(3):179-87. doi: 10.1177/0891988712458366. [PubMed: 23124012]

34. Kapoor WN, Karpf M, Wieand S, Peterson JR, Levey GS. A prospective evaluation and follow-up of patients with syncope. $N$ Engl $J$ Med. 1983;309(4):197-204. doi: 10.1056/NEJM198307283090401. [PubMed: 6866032]

35. Katon W, Kleinman A, Rosen G. Depression and somatization: a review. Part I. Am J Med. 1982;72(1):127-35. [PubMed: 7058818] 